\title{
Deep Brain Stimulation: A New Era in Functional Neuroscience? Laura Sperry ${ }^{1}$ and Lin Zhang ${ }^{1,2 *}$
}

${ }^{1}$ Department of Neurology, University of California, Davis, School of Medicine, Sacramento, CA, USA

${ }^{2}$ Neurological Surgery, University of California, Davis, School of Medicine, Sacramento, CA, USA

Deep Brain Stimulation (DBS) evolved from functional stereotactic neurosurgery techniques designed to produce selective lesions of the thalamus and cerebellum [1]. In 1952, Irving S. Cooper discovered the potential of ligation therapy when he unintentionally occluded the anterior choroidal artery, causing a medial globus pallidal infarction, which, surprisingly, alleviated rest tremor, rigidity and contralateral cogwheeling. Throughout the 1950s and 1960s, techniques for ablative procedures were refined with much focus on identifying the ideal targets. The motor thalamus and Gobus Pallidusinternus (GPi) were considered the most effective targets [2,3]. In the 1960s, L-dopa became the standard of care for Parkinson's disease (PD) and the popularity of ablative surgeries significantly decreased. During this time, ablative surgeries were primarily limited to thalamotomy to treat tremor and pallidotomy and thalamotomy for dystonia; they were rarely done to treat PD. However, by the 1980s, the limitations (motor fluctuations and dyskinesias) of L-dopa became apparent and ablative surgeries for PD regained popularity $[1,3,4]$. Building on the experience of ablative surgeries, stimulation procedures started to become commonplace in the management of movement disorders by the 1990s. This introduced a new era in functional neuroscience for movement disorders.

Over the last 20 years, DBS has become the surgical treatment of choice for movement disorders due to its superior safety profile over ablative procedures and the ability to adjust and potentially reverse the stimulation effects $[3,5,6]$. Since 1995, Medtronic reports over 80,000 individuals have been implanted stereotactically worldwide [7]. Despite its popularity, there is still controversy over the superiority of medical therapy versus DBS. Currently, PD and Essential Tremor (ET) are the only FDA approved indications for DBS, with dystonia and obsessive compulsive disorder being approved under a Humanitarian Device Exemption; however, many more neurological and psychiatric disorders are currently being studied $[7,8]$.

While the advent of DBS has revolutionized the field of functional neuroscience, it is still very much in its infancy. Beyond the currently approved neurological indications, our group has shown DBS to have significant impact in tremor control for patients with the neurodegenerative disorder, Fragile X-associated Tremor Ataxia Syndrome (FXTAS), where patients initially present with intention tremor followed by gait ataxia [9]. As technologies continue to advance, functional neuroscience will not only be the future of clinical neurology but likely will have a stronghold as an accepted treatment alternative for disease processes such as depression, epilepsy, eating disorder, cluster headaches, chronic and phantom limb pain, Tourette's syndrome, drug resistant hypertension and posttraumatic coma $[3,10]$.

The American Society for Stereotaxic and Functional Neurosurgery (ASSFN) recently held a research conference to discuss the future of neuromodulation, much of which focused on emerging applications of DBS (The Parkinson Alliance/DBS-STN Research Team, nd).Not only are there many studies focusing on the use of DBS to reduce seizure activity, there is a group with The Department of Neurosurgery at the Medical College of Georgia investigating the use of "responsive neurostimulation", where the DBS is triggered by intrinsic brain activity that precedes a seizure, thus, intervening before the seizure even starts.
Another emerging application is the use of DBS on neuroregulation of feeding behavior to target severe overeating disorders and morbid obesity. Currently, the research is being done on rodents, pigs and large animal models to demonstrate weight control with ventromedial hypothalamus DBS [11,12]. Further, while correct targeting is still being investigated, DBS appears to be offering new treatment options for patients with refractory neurologic and psychiatric diseases, such as Tourette's syndrome, depression and addictive behavior in patients with substance dependence $[1,13]$.

While there are still many unanswered questions regarding targeting, patient selection criteria, and timing of the procedure, the field of functional neuroscience, with DBS currently at the forefront, has positioned itself to be a considerable therapeutic option in a variety of refractory neurologic and psychiatric disorders [1]. As research progresses on drug resistant hypertension, it is conceivable that DBS will undoubtedly expand beyond these fields and become the flagship of functional neuroscience.

\section{References}

1. Sironi V.A. (2011) Origin and evolution of deep brain stimulation. Front Integr Neurosci, 5

2. Das K, Benzil DL, Rovit RL, Murali R, Couldwell WT (1998) Irving S. Cooper (1922-1985): a pioneer in functional neurosurgery. J Neurosurg 89: 865-873.

3. Rezai AR, Machado AG, Deogaonkar M, Azmi H, Kubu C, et al. (2008) Surgery for movement disorders. Neurosurgery 62: 809-38.

4. Rodriguez-Oroz MC, Obeso JA, Lang AE, Houeto JL, Pollak P, et al. (2005) Bilateral deep brain stimulation in Parkinson's disease: a multicentre study with 4 years follow-up. Brain 128: 2240-2249.

5. Benabid AL, Pollak P, Gervason C, Hoffmann D, Gao DM et al. (1991) Longterm suppression of tremor by chronic stimulation of the ventral intermediate thalamic nucleus. Lancet 337: 403-406.

6. Weaver FM, Follett K, Stern M, Hur K, Harris C, et al. (2009) Bilateral deep brain stimulation vs best medical therapy for patients with advanced Parkinson disease: a randomized controlled trial. JAMA 301:63-73.

7. Medtronic, Inc. (2012) Deep brain stimulation for movement disorders Retrieved February 25, 2013

8. Marks WJ Jr, (2011) Deep Brain Stimulation Management. Cambridge University Press, Cambridge UK.

9. Hagerman RJ, Pak J, Sperr, L, Ortigas M, Olichney J, et al. (2012) Case series: Deep brain stimulation in patients with Fragile X-Associated Tremor Ataxia syndrome (FXTAS). Brain Disorders and Therapy. Submitted.

*Corresponding author: Lin Zhang, M.D., Ph.D, Lawrence J Ellison Ambulatory Care Center 4860 Y St., Suite 3700 Sacramento, CA 95817, Phone: 916-7346280; Fax: (916) 734-6525, E-mail: lin.zhang@ucdmc.ucdavis.edu

Received February 27, 2013; Accepted March 01, 2013; Published March 04 2013

Citation: Sperry L, Zhang L (2013) Deep Brain Stimulation: A New Era in Functional Neuroscience? Brain Disord Ther 2: e105. doi:10.4172/2168-975X.1000e105

Copyright: $\odot 2013$ Sperry L, et al. This is an open-access article distributed under the terms of the Creative Commons Attribution License, which permits unrestricted use, distribution, and reproduction in any medium, provided the original author and source are credited. 
Citation: Sperry L, Zhang L (2013) Deep Brain Stimulation: A New Era in Functional Neuroscience? Brain Disord Ther 2: e105. doi:10.4172/2168975X.1000e105

Page 2 of 2

10. Ryszard MP, Gabriela DP, Robert MG (2011) Deep Brain Stimulation. JAMA 305: 732.

11. Melega WP, Lacan G, Gorgulho AA, Behnke EJ, De Salles AA (2012) Hypothalamic deep brain stimulation reduces weight gain in an obesity-animal model. PLoS One 7.
12. The Parkinson Alliance/DBS-STN Research Team. (nd) The Future of Neuromodulation. DBS-STN.org. (2013).

13. Kuhn J, Gründler TO, Lenartz D, Sturm V, Klosterkötter J, et al. (2010) Deep brain stimulation for psychiatric disorders. Dtsch Arztebl Int 107: 105-13. 